\title{
APLICAÇÃO DO MÉTODO KANITZ PARA VERIFICAÇÃO DE SOLVÊNCIA NAS EMPRESAS OI S/A E TIM S/A.
}

\section{APPLICATION OF THE KANITZ METHOD TO CHECK SOLVENCY IN OI S/A AND TIM S/A COMPANIES}

Flavio Heleno Solano Reis ${ }^{1}$, Petrus Fabiano Araújo de Oliveira², Josy Hellen Azevedo Braga ${ }^{3}$, Milton Pinheiro de Almeida Netto ${ }^{4}$, Mayla Rodrigues Barbosa Cunha ${ }^{5}$

Submetido: $16 / 02 / 2021$

Aprovado: 26/02/2021

\section{RESUMO}

Analisar as demonstrações financeiras de uma empresa detalhadamente, melhora a percepção do gestor para a tomada decisões. Esta pesquisa revela uma ferramenta que auxilia nessa análise e tem como objetivo identificar o grau de solvência ou insolvência de empresas do setor de telecomunicação através do método de Kanitz. O termômetro de Kanitz foi criado a partir de dados e experimentos estatísticos avaliando empresas e sua performance, sendo utilizado como ferramenta de gestão financeira para analisar as empresas. Esta pesquisa se classifica como quali-quanti e neste trabalho serão utilizados dados das empresas Tim S/A e Oi S/A, duas das principais empresas do setor de telecomunicações do Brasil. Os dados serão inseridos nas formulas do método, que resultarão nas informações necessárias e por fim indicarão a posição que cada entidade se encontra, a fim de gerar informações para que se possa fazer um gráfico para cada empresa, gerando assim informações para serem utilizadas no acompanhamento e gestão das mesmas, guardando as particularidades de cada empresa.

PALAVRAS-CHAVE: Método Kanitz. Solvência. Análise financeira.

\begin{abstract}
Analyzing a company's financial statements in detail, improves the manager's perception for decision making. This research reveals a tool that assists in this analysis and aims to identify the degree of solvency or insolvency of companies in the telecommunications sector through the Kanitz method. Kanitz's thermometer was created from data and statistical experiments evaluating companies and their performance, being used as a financial management tool to analyze companies. This research is classified as quali-quanti and this work will use data from the companies Tim S/A and Oi S/A, two of the main companies in the telecommunications sector in Brazil. The data will be inserted in the formulas of the method, which will result in the necessary information and finally indicate the position that each entity is in order to generate information so that a graph can be made for each company, thus generating information to be used in monitoring and management, keeping the particularities of each company.
\end{abstract}

KEYWORDS: Kanitz method. Solvency. Financial analysis.

\footnotetext{
${ }^{1}$ EGPA/Cosmopolita/ESMAC - fhsolano@gmail.com

2 Mestre em Administração, pela Universidade da Amazônia (UNAMA), Contador do Escritório Contábil Oliveira Contabilidade; Professor Universitário. - http://orcid.org/0000-0003-1032-7253 - petrusoliveira270@gmail.com

3 Pós-graduação em Contabilidade, Gestão Tributária e Auditoria; e MBA em Gestão Financeira, Auditoria e Controladoria ambas pela Faculdade Estratego; Coordenadora de Departamento Contábil. http://orcid.org/0000-0002-3939-8576 - josybraga41@gmail.com

${ }^{4}$ Bacharel em Ciências Contábeis - ESMAC - http://orcid.org/0000-0002-0471-3644 - milton_ntto@yahoo.com.br

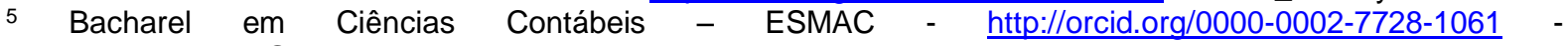
maylabarbosa.123@gmail.com
} 


\section{RECIMA21 - REVISTA CIENTÍFICA MULTIDISCIPLINAR}

APLICAÇÃO DO MÉTODO KANITZ PARA VERIFICAÇÃO DE SOLVÊNCIA NAS EMPRESAS OI S/A E TIM S/A.

Flavio Heleno Solano Reis, Petrus Fabiano Araújo de Oliveira, Josy Hellen Azevedo Braga, Milton Pinheiro de Almeida Netto, Mayla Rodrigues Barbosa Cunha

\section{INTRODUÇÃO}

A origem da contabilidade surgiu da necessidade do homem fazer os seus registros de entrada e saída. A contabilidade está "voltada para a melhor utilização dos custos econômicos da empresa, por meio de adequado controle dos insumos, efetuado por um sistema de informação gerencial "(CREPALDI, 2006, p. 16).

Nesta pesquisa é abordada uma ferramenta de auxílio na análise das demonstrações financeiras: o termômetro de Kanitz. E como o mesmo pode atuar na indicação de solvência ou insolvência das empresas de telecomunicações OI S/A e TIM S/A.

Segundo o site WALD, a empresa OI S/A requereu o pedido de recuperação judicial em 20 de junho de 2016, tendo como base lei de recuperação judicial e falências (Lei no 11.101/2005). A empresa TIM S/A encontra-se em situação aparentemente estável em relação a suas finanças. Esta pesquisa se propõe a comparar as duas empresas e seus demonstrativos financeiros entre os períodos selecionados.

O artigo tem como objetivo geral identificar o grau de solvência ou insolvência de empresas do setor de telecomunicação através da aplicação do método de Kanitz, e ainda se propõe: descrever o método de Kanitz; levantar as informações financeiras geradas pelo método Kanitz para as empresas Tim S/A e OI S/A; demonstrar a funcionalidade do método, como ferramenta de análise.

\subsection{Fundamentação Teórica}

\subsubsection{Contabilidade}

A contabilidade inicialmente era desenvolvida apenas por donos de patrimônio, porém com o decorrer do tempo, novos atuantes passaram a utilizar, estes eram: fornecedores de mercadorias, o governo, administradores, acionistas, empregados, clientes, e sindicatos entre outros.

Segundo Marion (2002, p. 128), "a contabilidade é uma ciência social, pois estuda o comportamento das riquezas que se integram no patrimônio, em face das ações humanas".

\subsubsection{Contabilidade Gerencial}

A contabilidade gerencial, está inserida dentro da contabilidade e, é o mecanismo utilizado pela administração da empresa para colher as informações contábeis e manter a estabilidade da entidade, 


\section{RECIMA21 - REVISTA CIENTÍFICA MULTIDISCIPLINAR}

APLICAÇÃO DO MÉTODO KANITZ PARA VERIFICAÇÃO DE SOLVÊNCIA NAS EMPRESAS OI S/A E TIM S/A.

Flavio Heleno Solano Reis, Petrus Fabiano Araújo de Oliveira, Josy Hellen Azevedo Braga, Milton Pinheiro de Almeida Netto, Mayla Rodrigues Barbosa Cunha

Conforme ludícibus (1998, p. 21) descreve:

"A contabilidade gerencial, num sentido mais profundo, está voltada única e exclusivamente para a administração da empresa, procurando suprir informações que se "encaixem" de maneira válida e efetiva no modelo decisório do administrador".

Dessa forma, a contabilidade gerencial é vista como ferramenta importante para o processo de tomada de decisão.

\subsubsection{Demonstrações Financeiras}

Demonstrações Financeiras são documentos elaborados e fornecidos pela contabilidade da empresa, e compreende todas as ocorrências contábeis desta entidade em um determinado período ou mesmo no final do ano em exercício. Entre as demonstrações financeiras temos: Balanço Patrimonial e DRE - Demonstração de Resultado do exercício.

\section{Quadro 1: Demonstrações Contábeis}

\begin{tabular}{|c|l|}
\hline Balanço Patrimonial & $\begin{array}{l}\text { Área onde estão descritas todas as movimentações de patrimônio da empresa. Estas } \\
\text { movimentações são divididas em três subgrupos: Ativo, Passivo e Patrimônio Líquido. }\end{array}$ \\
\hline $\begin{array}{c}\text { Demonstração de } \\
\text { Resultado do exercício }\end{array}$ & $\begin{array}{l}\text { Onde encontra-se o resultado obtido pela empresa em um determinado período, ou } \\
\text { seja, lucro ou prejuízo. Nesta área são registradas todas as receitas, despesas e } \\
\text { custos da entidade. }\end{array}$ \\
\hline
\end{tabular}

Fonte: Adaptado de Diniz (2015).

\subsubsection{Indicadores de Investimento}

Os principais indicadores de investimento são os índices de liquidez, rentabilidade e endividamento. Para Marion (2006, p. 15) "Só teremos condições de conhecer a situação econômicofinanceira de uma empresa por meio de três pontos fundamentais de análise: liquidez (situação financeira), rentabilidade (situação econômica) e endividamento (estrutura de capital)".

\subsubsection{1 Índices de Liquidez}

Através de dados contábeis os índices de liquidez fornecem resultados que retratam a situação financeira da empresa.

Almeida (2019) retrata que "Índices de liquidez contemplam relacionamentos entre contas do Balanço Patrimonial e demonstram a capacidade da entidade de honrar os seus compromissos financeiros". 


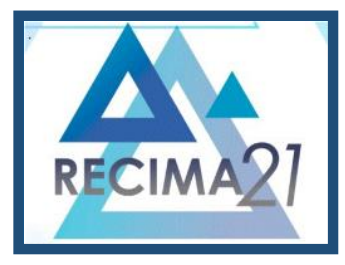

\section{RECIMA21 - REVISTA CIENTÍFICA MULTIDISCIPLINAR}

APLICAÇÃO DO MÉTODO KANITZ PARA VERIFICAÇÃO DE SOLVÊNCIA NAS EMPRESAS OI S/A E TIM S/A.

Flavio Heleno Solano Reis, Petrus Fabiano Araújo de Oliveira, Josy Hellen Azevedo Braga, Milton Pinheiro de Almeida Netto, Mayla Rodrigues Barbosa Cunha

O índice em questão é dividido em quatro índices, conforme tabela a seguir:

Quadro 2: Índices de liquidez

\begin{tabular}{|c|l|}
\hline Liquidez Corrente (LC) & $\begin{array}{l}\text { Mede a capacidade que uma entidade tem de pagar suas contas a curto } \\
\text { prazo, ou seja, em um mesmo exercício financeiro. }\end{array}$ \\
\hline Liquidez Imediata (LI) & $\begin{array}{l}\text { Relata a condição da empresa em arcar com seus compromissos a curto } \\
\text { prazo, dispondo apenas de recursos que poderão ser imediatamente } \\
\text { disponíveis (caixa, banco e investimento de curtíssimo prazo) }\end{array}$ \\
\hline Liquidez seca (LS) & $\begin{array}{l}\text { Indica a capacidade que a empresa tem de pagar suas dívidas a curto } \\
\text { prazo desconsiderando seu estoque. }\end{array}$ \\
\hline Liquidez geral (LG) & $\begin{array}{l}\text { Mostra a capacidade que a empresa tem de quitar suas dívidas em longo } \\
\text { prazo, levando em consideração tudo que a empresa dispõe para converter } \\
\text { em dinheiro a curto, médio e longo prazo. }\end{array}$ \\
\hline
\end{tabular}

Fonte: Adaptado de Diniz (2015).

\subsubsection{2 Índice de Endividamento}

Através do índice de endividamento é possível visualizar se a empresa é composta em sua maioria de capital próprio ou de terceiros.

Segundo Marion (2002, p.646), índice de endividamento é "uma participação de capital de terceiros exagerada em relação ao capital próprio torna a empresa vulnerável a qualquer intempérie".

\subsubsection{3 Índice de Rentabilidade}

O índice de rentabilidade é uma ferramenta importante para demonstrar o desempenho dos negócios. Segundo Almeida (2019), o índice de rentabilidade demonstra o retorno em termos do lucro auferido pela entidade em comparação com receitas, ativos, capitais próprios (patrimônio líquido) e outros parâmetros.

\subsubsection{Termômetro de Kanitz}

Segundo Kassai e Kassai (2003, p 1), o termômetro de Kanitz é a ferramenta utilizada para medir a solvência ou insolvência de uma empresa. Este método foi desenvolvido na década de 70 pelo professor Stephen Charles Kanitz com base em uma análise crítica. 


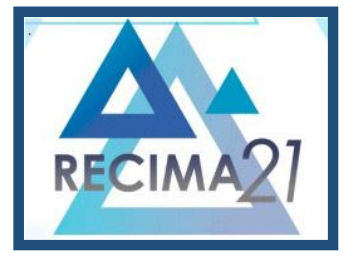

\section{RECIMA21 - REVISTA CIENTÍFICA MULTIDISCIPLINAR}

APLICAÇÃO DO MÉTODO KANITZ PARA VERIFICAÇÃO DE SOLVÊNCIA NAS EMPRESAS OI S/A E TIM S/A.

Flavio Heleno Solano Reis, Petrus Fabiano Araújo de Oliveira, Josy Hellen Azevedo Braga, Milton Pinheiro de Almeida Netto, Mayla Rodrigues Barbosa Cunha

Para chegar ao resultado de solvência ou insolvência é necessário extrair alguns dados do Balanço Patrimonial e da DRE, em seguida aplicar os mesmos nas fórmulas dos índices de liquidez, rentabilidade e endividamento, e por fim aplicá-los na fórmula elaborada por Kanitz.

\subsubsection{Método de Kanitz}

Stephen C. Kanitz desenvolveu um método capaz de prever a falência em empresas através dos dados financeiros de empresas que realmente faliram.

A partir dessas análises e de estudos estatísticos, Kanitz chegou a um termo denominado Fator de Insolvência. Também chamado de Termômetro de Insolvência, o mesmo tem uma escala que varia entre os valores (-) 7,0 a 7,0.

Se a nota for positiva, ou seja, variando de 0 a 7,0 pode se concluir que existe uma solvência, um equilíbrio na estrutura financeira da empresa, sendo tanto maior quanto o resultado se aproximar do número 7,0. (Oliveira, 2019)

Se a nota for de 0 a (-) 3,0, existe uma situação indefinida e com indícios de falência pois essa região é chamada de penumbra. Se esse resultado estiver entre: (-) 3,0 e (-) 7,0, a propensão de falência dessa empresa é alta, ela está em um estado de insolvência segundo o método, como mostra a figura abaixo:

Figura 1: Termômetro de Insolvência de Kanitz

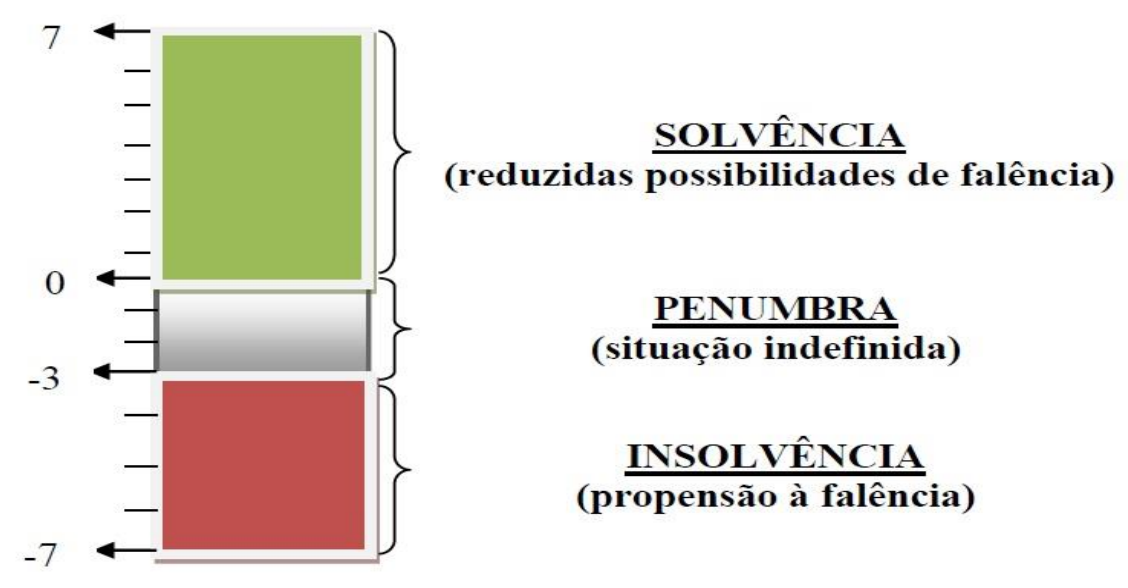

Fonte: Extraído de Oliveira (2019).

A fórmula criada por Kanitz, que consiste no modelo abaixo para chegar a esses resultados, consiste nos seguintes dados: 


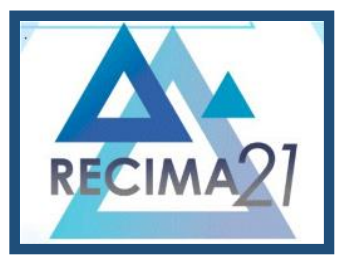

\section{RECIMA21 - REVISTA CIENTÍFICA MULTIDISCIPLINAR}

APLICAÇÃO DO MÉTODO KANITZ PARA VERIFICAÇÃO DE SOLVÊNCIA NAS EMPRESAS OI S/A E TIM S/A.

Flavio Heleno Solano Reis, Petrus Fabiano Araújo de Oliveira, Josy Hellen Azevedo Braga, Milton Pinheiro de Almeida Netto, Mayla Rodrigues Barbosa Cunha

Fator de Insolvência $=0,05^{*} \mathrm{X} 1+1.65 * \mathrm{X} 2+3.55^{\star} \mathrm{X} 3-1.06{ }^{*} \mathrm{X} 4-0.33^{*} \mathrm{X} 5$

Onde $\mathrm{X} 1, \mathrm{X} 2, \mathrm{X} 3, \mathrm{X} 4$ e X5 são respectivamente como segue no quadro abaixo:

Quadro 3: Variáveis do Termômetro de Kanitz

\begin{tabular}{|c|c|c|}
\multicolumn{2}{|c|}{ ÍNDICES } & FÓRMULAS \\
\hline X1 & Índice de Retorno do Patrimônio Líquido & $\frac{\text { Lucro liquido }}{\text { Patrimonio liquido }}$ \\
\hline X2 & Índice de Liquidez Geral & $\frac{\text { Ativo Circulante + Realizavel a longo prazo }}{\text { Passivo Circulante + Passivo não Circulante }}$ \\
\hline X3 & Índice de Liquidez Seca & $\frac{\text { Ativo Circulante - Estoque }}{\text { Pasivo Circulante }}$ \\
\hline X4 & Índice de Liquidez Corrente & $\frac{\text { Ativo Circulante }}{\text { Pasivo Circulante }}$ \\
\hline X5 & Índice de Participação de Capitais de Terceiros & $\frac{\text { Passivo Circulante + Passivo não Circulante }}{\text { Patrimonio Liquido }}$ \\
\hline
\end{tabular}

Fonte: Extraído de Oliveira (2019).

Observação: Como as empresas são do setor de serviços, e portanto, não tem estoque para cálculo da liquidez seca, foi feita uma adaptação para chegar ao resultado da liquidez seca, será a fórmula de Almeida (2019), abaixo para o cálculo:

$$
\text { Liquidez seca }=\frac{D+C R+A F}{P C}
$$

Onde, $\mathrm{D}=$ disponível $; \mathrm{CR}=$ contas a receber $; \mathrm{AF}=$ aplicações financeiras $\mathrm{e}$

$$
\mathrm{PC}=\text { passivo circulante }
$$

\subsubsection{Contas Utilizadas}

As contas das empresas que serão utilizadas na pesquisa são as seguintes: Lucro Líquido; Patrimônio Líquido; Ativo Circulante; Realizável a Longo Prazo; Passivo Circulante; Passivo não Circulante; Estoque; e também as contas: Disponível; Contas a Receber e; Aplicações Financeiras.

\section{MATERIAL E MÉTODOS}




\section{RECIMA21 - REVISTA CIENTÍFICA MULTIDISCIPLINAR}

APLICAÇÃO DO MÉTODO KANITZ PARA VERIFICAÇÃO DE SOLVÊNCIA NAS EMPRESAS OI S/A E TIM S/A.

Flavio Heleno Solano Reis, Petrus Fabiano Araújo de Oliveira, Josy Hellen Azevedo Braga, Milton Pinheiro de Almeida Netto, Mayla Rodrigues Barbosa Cunha

Descreveremos o tratamento e a utilização das contas das empresas para posterior aplicação no termômetro de insolvência.

A presente pesquisa parte da aplicação da teoria desenvolvida pelo professor Stephen Charles Kanitz em dados contábeis das empresas TIM S/A e OI S/A, com isso pode-se classificar este estudo como quali-quantitativo, pois segundo Farias Filho e Arruda Filho (2015, p. 63-64) o processo da pesquisa qualitativa parte da interpretação dos fenômenos e a atribuição dos significados, já a quantitativa traduz em números opiniões e informações para classificá-las e analisálas.

Primeiramente serão feitos levantamentos bibliográficos e, documentais, ou seja, o estudo do método com base em teorias e em seguida seleção de algumas contas do Balanço Patrimonial e da DRE das empresas do setor de telecomunicação em questão. Para Gil (2002, p.45) procedimentos bibliográficos e documentais assemelham-se muito.

"A diferença essencial entre ambas está na natureza das fontes. Enquanto a pesquisa bibliográfica se utiliza fundamentalmente das contribuições dos diversos autores sobre determinado assunto, a pesquisa documental vale-se de materiais que não recebem ainda um tratamento analítico, ou que ainda podem ser reelaborados de acordo com os objetos da pesquisa".

Para Farias Filho e Arruda Filho (2015, p. 63) pesquisa exploratória visa proporcionar maior familiaridade com o problema, ou seja, realização de levantamentos bibliográficos, busca de web, entre outros e pesquisa descritiva visa descrever as caraterísticas de determinada população ou fenômeno. Diante disto, este trabalho classifica-se como exploratório e descritivo, pois houve a necessidade de buscar bases teóricas em livros, internet, artigos e outros, do tema tratado e posteriormente, descrever de forma detalhada, a construção do método estudado como ferramenta de uso nas organizações.

O período utilizado para esse estudo de caso será longitudinal de 05 anos consecutivos, mais especificamente de 2014 a 2018. De acordo com Farias Filho e Arruda Filho (2015, p.62) estudos longitudinais são estudos realizados em um intervalo qualquer de tempo.

\section{ANALISE E RESULTADOS}

\subsection{Comparativo de Dados}

Conforme tabela 1 e 2 abaixo, pode-se observar quais contas presentes no sistema contábil serão utilizadas no desenvolvimento do primeiro passo da aplicação do método que está sendo desenvolvido na pesquisa.

Tabela 1: Dados OI S/A (Valores em milhares de reais) 


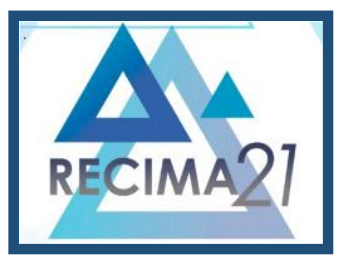

\section{RECIMA21 - REVISTA CIENTÍFICA MULTIDISCIPLINAR}

APLICAÇÃO DO MÉTODO KANITZ PARA VERIFICAÇÃO DE SOLVÊNCIA NAS EMPRESAS OI S/A E TIM S/A

Flavio Heleno Solano Reis, Petrus Fabiano Araújo de Oliveira, Josy Hellen Azevedo Braga, Milton Pinheiro de Almeida Netto, Mayla Rodrigues Barbosa Cunha

\begin{tabular}{l|c|c|c|c|c}
\hline \multicolumn{1}{c}{ Contas } & $\mathbf{2 0 1 4}$ & $\mathbf{2 0 1 5}$ & $\mathbf{2 0 1 6}$ & $\mathbf{2 0 1 7}$ \\
\hline Ativo Circulante & 49.286 .795 & 38.067 .009 & 26.211 .728 & 23.747 .748 & 21.313 .484 \\
\hline Disponível (caixa e Eq. Caixa) & 2.449 .206 & 14.898 .063 & 7.563 .251 & 6.862 .684 & 4.385 .329 \\
\hline Aplicações financeiras & 171.415 & 1.801 .720 & 116.532 & 21.447 & 201.975 \\
\hline Contas a Receber & & & & \\
\hline Realizável a Longo Prazo & 7.450 .040 & 8.379 .719 & 7.891 .078 & 7.367 .442 & 6.516 .555 \\
\hline Passivo Circulante & 23.992 .912 & 29.993 .945 & 9.583 .225 & 9.415 .446 & 8.632 .464 \\
\hline Passivo Não Circulante & & & & \\
\hline Patrimônio Liquido & 42.556 .832 & 25.574 .071 & 61.285 .760 & 67.891 .607 & 10.689 .459 \\
\hline Lucro/prejuízo Liquido & 40.920 .801 & 57.034 .524 & 11.352 .264 & 14.259 .952 & 31.852 .527 \\
\hline
\end{tabular}

Fonte: Extraído de Relatórios Anuais OI.

Tabela 2: Dados TIM S/A (Valores em milhares de reais)

TIM

\begin{tabular}{|c|c|c|c|c|c|}
\hline Contas & 2014 & 2015 & 2016 & 2017 & 2018 \\
\hline Ativo Circulante & 11.174 .415 & 12.040 .246 & 10.107 .399 & 7.607 .388 & 5.998 .126 \\
\hline Disponível (caixa e Eq. Caixa) & 5.232 .992 & 6.100 .403 & 5.128 .186 & 2.960 .718 & 1.075 .530 \\
\hline Aplicações financeiras & - & - & - & - & - \\
\hline Contas a Receber & 3.537 .417 & 2.858 .089 & 2.919 .177 & 2.540 .856 & 2.838 .808 \\
\hline Realizável a Longo Prazo & 2.931 .166 & 2.889 .601 & 2.831 .176 & 2.841 .962 & 4.074 .137 \\
\hline Passivo Circulante & 9.123 .256 & 9.166 .864 & 7.281 .555 & 7.224 .437 & 7.075 .379 \\
\hline Passivo Não Circulante & 7.987 .854 & 9.812 .202 & 10.186 .612 & 7.224 .744 & 5.087 .673 \\
\hline Patrimônio Liquido & 15.322 .034 & 16.577 .322 & 17.187 .513 & 18.151 .184 & 19.794 .837 \\
\hline Lucro/prejuízo Liquido & 1.546 .419 & 2.085 .442 & 750.427 & 1.234 .507 & 2.545 .101 \\
\hline
\end{tabular}

Fonte: Extraído de Demonstrações Financeiras TIM. 


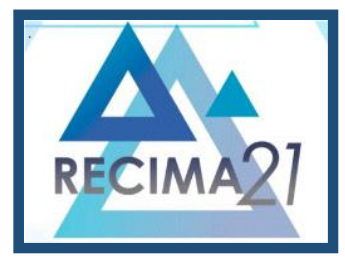

\section{RECIMA21 - REVISTA CIENTÍFICA MULTIDISCIPLINAR}

APLICAÇÃO DO MÉTODO KANITZ PARA VERIFICAÇÃO DE SOLVÊNCIA NAS EMPRESAS OI S/A E TIM S/A. Flavio Heleno Solano Reis, Petrus Fabiano Araújo de Oliveira, Josy Hellen Azevedo Braga, Milton Pinheiro de Almeida Netto, Mayla Rodrigues Barbosa Cunha

Diante das informações presentes na tabela 1 pode-se verificar que entre os períodos de 2014 a 2017 a empresa Ol teve prejuízos financeiros crescentes, conseguindo um lucro líquido apenas no ano de 2018, período em que foi homologada sua recuperação judicial.

Já na tabela 02, observa se que a empresa TIM possui resultados financeiros estáveis, apesar de não possuir aplicações financeiras diretas, tendo um crescente lucro nos anos de 2014 e 2015; uma pequena queda no ano de 2016, mas voltando a ter crescimento nos períodos de 2017 e 2018.

\subsection{Comparativo dos Índices}

Diante das informações coletadas, foi possível o início do desenvolvimento do método, que parte da aplicação dos dados financeiros nas fórmulas que compõe X1 a X5 das tabelas 3 e 4 . Vale ressaltar que tais fórmulas irão revelar os índices de endividamento, liquidez e rentabilidade.

Portanto, para melhor entendimento dos resultados que serão expostos nas tabelas 3 e 4 , deve ser entendido que todos os índices desenvolvidos devem ter resultado superior a 1 para possa significar positividade, pois contabilmente demonstraria que a cada $R \$ 1,00$ real de obrigação, a empresa deve dispor de ao menos $R \$ 1,00$ real de bens ou diretos afim de que possa honrar com suas dívidas.

Os índices de liquidez representados por X2, X3 e X4, nas tabelas 3 e 4, que correspondem aos índices de liquidez geral, seca e corrente respectivamente, demonstram a situação da empresa quanto ao comprimento de suas obrigações, levando em consideração seus disponíveis a longo prazo, imediato e a curto prazo. Por fim, o índice de endividamento representado por X5, nas referidas tabelas 3 e 4 , possibilita o gestor saber se sua empresa está dispondo em sua maioria de capital de terceiros ou de capital próprio. Diante do exposto podemos observar os resultados obtidos nas tabelas abaixo:

Tabela 3: Índices do Termômetro de Kanitz relativos à empresa OI

\begin{tabular}{c|c|c|c|c|c}
\hline $\mathbf{X}$ INDICES OI & $\mathbf{2 0 1 4}$ & $\mathbf{2 0 1 5}$ & $\mathbf{2 0 1 6}$ & $\mathbf{2 0 1 7}$ & $\mathbf{2 0 1 8}$ \\
\hline $\mathbf{X 2}$ & $-0,23$ & $-0,37$ & $-1,20$ & 0,47 & 1,08 \\
\hline $\mathbf{X 3}$ & 0,88 & 0,82 & 0,49 & 0,40 & 0,70 \\
\hline $\mathbf{X 4}$ & 0,24 & 0,98 & 0,25 & 0,21 & 1,04 \\
\hline $\mathbf{X 5}$ & 4,16 & 1,49 & 0,43 & 0,35 & 1,99 \\
\hline & & & & & \\
\hline
\end{tabular}

Fonte: Elaborado pelos Autores. 


\section{RECIMA21 - REVISTA CIENTÍFICA MULTIDISCIPLINAR}

APLICAÇÃO DO MÉTODO KANITZ PARA VERIFICAÇÃO DE SOLVÊNCIA NAS EMPRESAS OI S/A E TIM S/A. Flavio Heleno Solano Reis, Petrus Fabiano Araújo de Oliveira, Josy Hellen Azevedo Braga, Milton Pinheiro de Almeida Netto, Mayla Rodrigues Barbosa Cunha

Tabela 4: Índices do Termômetro de Kanitz relativos à empresa TIM

\begin{tabular}{c|c|c|c|c|c}
\hline INDICES TIM & $\mathbf{2 0 1 4}$ & $\mathbf{2 0 1 5}$ & $\mathbf{2 0 1 6}$ & $\mathbf{2 0 1 7}$ & $\mathbf{2 0 1 8}$ \\
\hline $\mathbf{X 1}$ & 0,10 & 0,13 & 0,04 & 0,07 & 0,13 \\
\hline $\mathbf{X 2}$ & 0,82 & 0,79 & 0,74 & 0,72 & 0,83 \\
\hline $\mathbf{X 3}$ & 0,96 & 0,98 & 1,11 & 0,76 & 0,55 \\
\hline $\mathbf{X 4}$ & 1,22 & 1,31 & 1,39 & 1,05 & 0,85 \\
\hline $\mathbf{X 5}$ & 1,12 & 1,14 & 1,02 & 0,80 & 0,61 \\
\hline
\end{tabular}

Fonte: Elaborado pelos Autores.

Pode-se observar que ambas empresas têm X1 abaixo do esperado, porém em situação crescente. Apenas em 2018 a Empresa OI encontra-se em situação positiva.

Em X2 as duas entidades encontram-se em situação negativa e regressiva, porém indicam uma recuperação em 2018. Já em X3 podemos notar que a empresa OI oscila com seus resultados, no entanto gerando saldo positivo em 2018; já a empresa TIM demonstra progressividade nos anos de 2014 e 2015, positividade em 2016 e uma queda considerável nos seguintes anos. No resultado de X4 observa-se que a empresa OI se encontra negativa apenas em 2016 e 2017 e a empresa TIM apenas em 2018.

Por fim em X5 é possível notar que a empresa OI tem valores crescentes até o ano de 2016, tendo uma enorme queda em 2017, demonstrando recuperação em 2018, a empresa TIM por outro lado, encontra-se negativa em 2017 e 2018.

\subsection{Comparativo de Insolvência}

Com todas as etapas que antecedem o desenvolvimento da fórmula do fator de insolvência concluídas, foram aplicados os resultados obtidos na mesma, gerando por fim os índices de insolvência das empresas correspondentes a cada ano que são mostrados na tabela abaixo, e, da mesma forma posteriormente através de gráficos, para melhor visualização dos resultados obtidos neste trabalho. Conforme tabela 05 abaixo e seus respectivos gráficos 1 e 2 :

Tabela 5: Fator de Insolvência das empresas OI e TIM

\begin{tabular}{c|c|c|c|c|c}
\hline PERIODO & $\mathbf{2 0 1 4}$ & $\mathbf{2 0 1 5}$ & $\mathbf{2 0 1 6}$ & $\mathbf{2 0 1 7}$ & $\mathbf{2 0 1 8}$ \\
\hline FATOR DE INSOLVENCIA OI & $-0,38$ & 1,35 & $-2,39$ & 3,07 & 2,18 \\
\hline
\end{tabular}




\section{RECIMA21 - REVISTA CIENTÍFICA MULTIDISCIPLINAR}

APLICAÇÃO DO MÉTODO KANITZ PARA VERIFICAÇÃO DE SOLVÊNCIA NAS EMPRESAS OI S/A E TIM S/A.

Flavio Heleno Solano Reis, Petrus Fabiano Araújo de Oliveira, Josy Hellen Azevedo Braga, Milton Pinheiro de Almeida Netto, Mayla Rodrigues Barbosa Cunha

\begin{tabular}{l|l|l|l|l|l}
\hline FATOR DE INSOLVENCIA TIM & 3,11 & 3,00 & 3,34 & 2,52 & 2,24 \\
\hline
\end{tabular}

Fonte: Elaborado pelos Autores.

Pode-se perceber através dos dados que compõem a tabela 5 que a empresa Ol esteve em situação de penumbra nos anos de 2014 e 2016, muito próximo da situação de insolvência. Para Marion (2019, p.117) o índice que se encontra "Entre (-) 3,0 e zero, é uma região nebulosa, e a empresa pode tanto sair da situação difícil ou pode falir" ou seja, este é o momento que os gestores devem revisar suas estratégias. Porém nos anos de 2015, 2017 e 2018 a mesma teve sua situação econômica solvente. Já a empresa TIM se manteve em todos os anos estudados solvente, porém sinalizando queda nos últimos anos.

Logo abaixo podemos visualizar os mesmos dados da tabela $5 \mathrm{em}$ forma de gráfico, dando melhor visualização dos resultados do fator de solvência obtidos pelas empresas TIM e OI nos anos de 2014 a 2018:

Gráfico 1: Fator de Insolvência OI

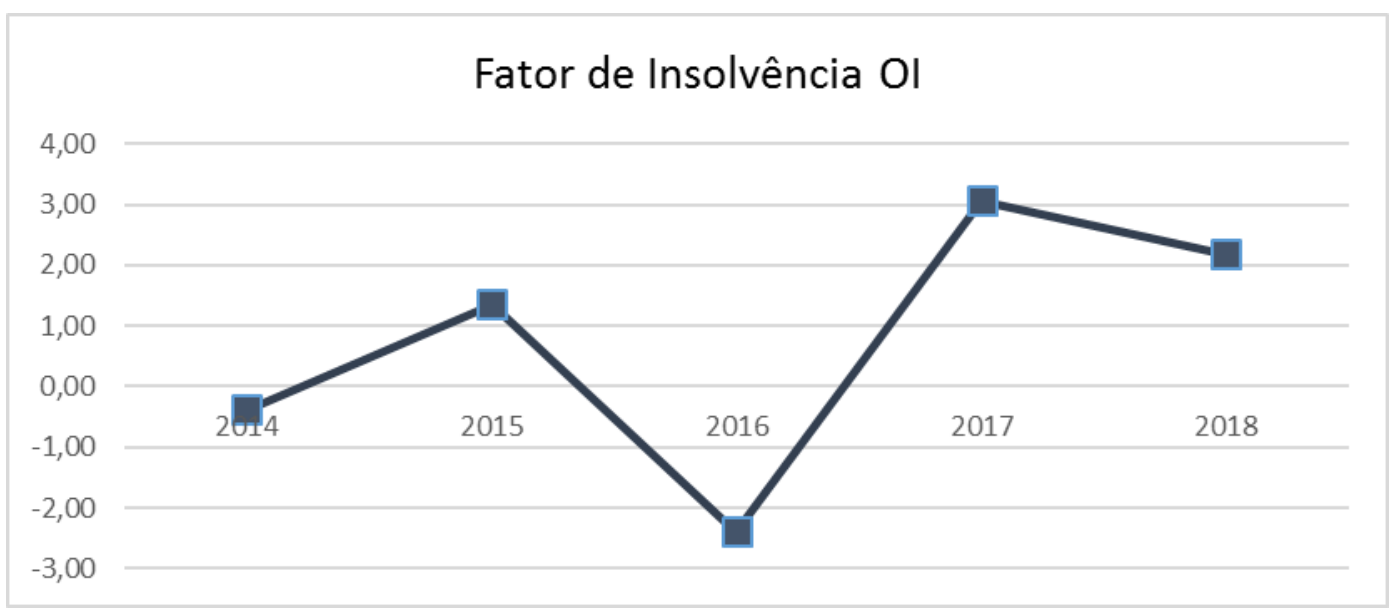

Fonte: Elaborado pelos Autores.

Gráfico 2: Fator de Insolvência TIM 


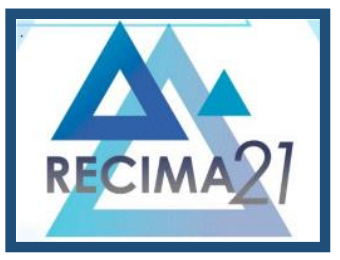

\section{RECIMA21 - REVISTA CIENTÍFICA MULTIDISCIPLINAR}

APLICAÇÃO DO MÉTODO KANITZ PARA VERIFICAÇÃO DE SOLVÊNCIA NAS EMPRESAS OI S/A E TIM S/A. Flavio Heleno Solano Reis, Petrus Fabiano Araújo de Oliveira, Josy Hellen Azevedo Braga, Milton Pinheiro de Almeida Netto, Mayla Rodrigues Barbosa Cunha

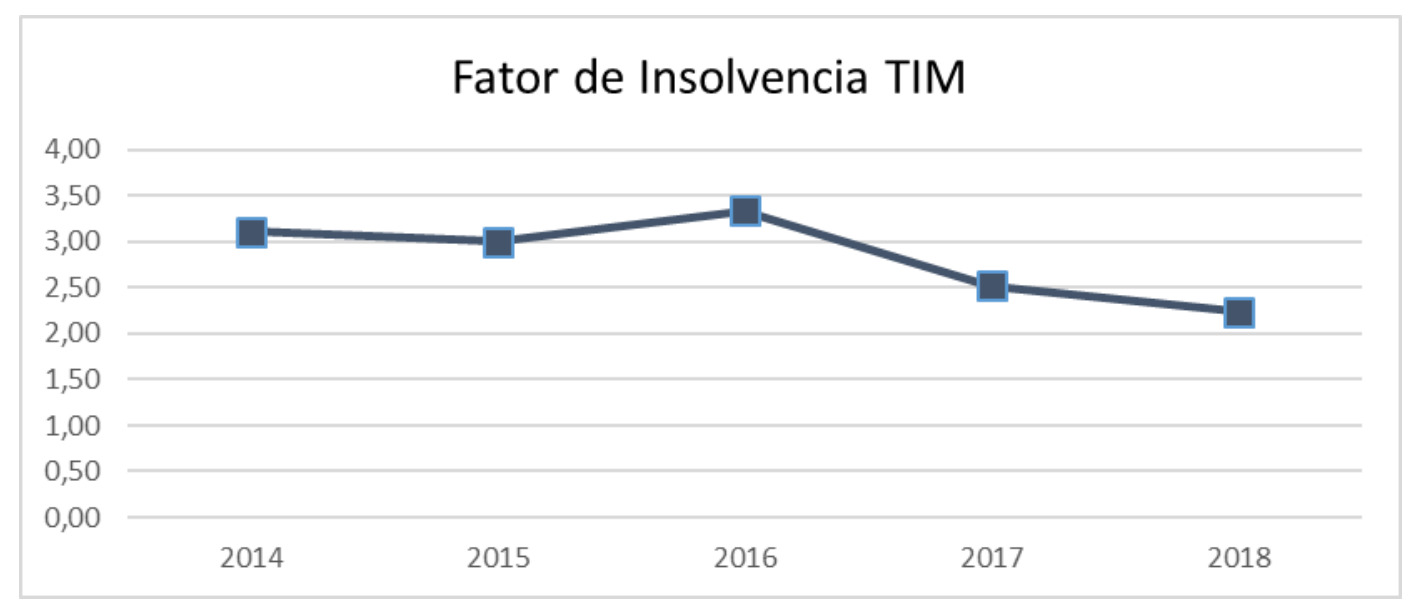

Fonte: Elaborado pelos Autores.

Como pode-se verificar os anos em que a empresa Ol esteve em situação de penumbra, ou seja, abaixo de zero e maior que menos três, foram os anos no qual a mesma iniciava sua recuperação judicial.

Pode-se verificar também a estabilidade citada anteriormente em relação a empresa TIM, tendo uma suave queda nos anos de 2017 e 2018, não obstante, conseguindo uma manutenção positiva de seus demonstrativos, mostrando que a empresa teve plena capacidade de honrar seus compromissos e obrigações e ainda gerar lucro através de uma boa administração de seus bens, direitos e obrigações.

\section{CONSIDERAÇÕES FINAIS}

O método de Stephen Charles Kanitz pode ser utilizado para verificar a situação económicofinanceira das empresas em geral, desde que as mesmas tenham uma contabilidade adequada segundo as Normas Brasileiras de Contabilidade e as leis vigentes no pais tais como a 6.404/76 (lei das Sociedade Anônima); a lei 123/2006 (lei das micro empresas e empresas de pequeno porte), dentre outras.

Este método tem como finalidade prever a capacidade que a empresa tem de cumprir com seus compromissos financeiros, ou seja, a sua solvência ou insolvência. A empresa Tim S/A que iniciou suas atividades no Brasil em 1998 e vem se mantendo no mercado, possuía em 2013, segundo dados da Bolsa de Valores de São Paulo - BOVESPA um lucro líquido de $R \$ 2.083 .781,82$ e obteve no primeiro semestre de 2019 um lucro líquido de $R \$ 4.107 .568,00$ mostrando um crescimento de 197,12\% ao longo dos sete anos (BOVESPA, 2019a); a empresa Oi S/A, por outro lado, está em processo de recuperação judicial, e teve um lucro líquido de $\mathrm{R} \$ 2.066 .344,70$ no ano 


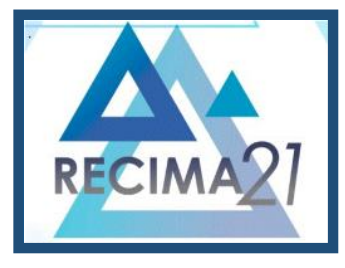

\section{RECIMA21 - REVISTA CIENTÍFICA MULTIDISCIPLINAR}

APLICAÇÃO DO MÉTODO KANITZ PARA VERIFICAÇ̃̃O DE SOLVÊNCIA NAS EMPRESAS OI S/A E TIM S/A.

Flavio Heleno Solano Reis, Petrus Fabiano Araújo de Oliveira, Josy Hellen Azevedo Braga, Milton Pinheiro de Almeida Netto, Mayla Rodrigues Barbosa Cunha

de 2013; porém no primeiro semestre do ano de 2019 obteve um prejuízo líquido de $R \$$ 5.708.166,00, portanto, tendo uma queda de 276,24\% (BOVESPA, 2019b).

A pesquisa teve como principal objetivo verificar a solvência ou insolvência das empresas OI S/A e TIM S/A objetivando um comparativo entre as duas empresas e demonstração do método de Kanitz.

O método de Kanitz tem como produto principal um fator de insolvência, no qual mostra em que nível de solvência ou insolvência a empresa está auxiliando os gestores sobre como estão seus demonstrativos financeiros e impulsionando os mesmos a tomar medidas no caso de uma situação desfavorável.

O método, para alcançar a meta proposta, necessita acessar os dados financeiros e contábeis das empresas, tabular os dados que serão utilizados, relacionar esses dados específicos entre si, gerando a partir desses resultados, índices, e, a partir disso, inserir os índices na fórmula de Kanitz, que elencam variáveis obtidas através de dados estatísticos pelo mesmo, gerando um número chamado de fator de insolvência que será colocado numa região especifica positiva, neutra ou negativa, que demonstrara a situação especifica da empresa naquele período.

Foram utilizados como ferramenta de pesquisa, os dados contábeis das respectivas empresas, que se encontram em seu domínio na internet. A pesquisa desenvolvida foi do tipo qualiquantitativa, pois foi necessário realizar um estudo aprofundado sobre a situação financeira de cada empresa, além de verificação de seus demonstrativos; e longitudinal com o período de cinco anos consecutivos, para análise dos dados contábeis das duas empresas atuantes do ramo de telecomunicações.

Por meio desse estudo, pode-se constatar o funcionamento do método, as situações financeiras indicadas pelo resultado da pesquisa em cada uma das empresas, e como o método pode ser um importante auxiliador na tomada de decisão dos gestores das empresas de telecomunicações.

Pode-se afirmar que a empresa Oi S/A teve momentos ruins, no período de tempo de 2014 a 2016, e no período de 2017, quando foi aprovada sua recuperação judicial e a empresa conseguiu parcelar seus débitos conforme acordado com os credores e o governo, conseguindo portanto que seus demonstrativos começassem a melhorar segundo aponta o método de Kanitz. Entre 2016 e 2017 a empresa sai da região de penumbra do termômetro e passa a região de solvência com um fator de 3,07 em 2017, tendo uma leve queda no ano de 2018, mas mostrando que a recuperação judicial foi de suma importância para a mudança nos números da empresa. 


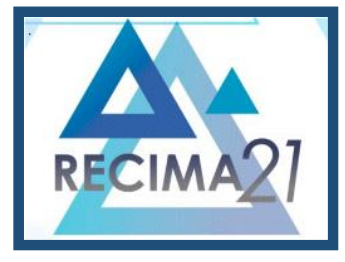

\section{RECIMA21 - REVISTA CIENTÍFICA MULTIDISCIPLINAR}

APLICAÇÃO DO MÉTODO KANITZ PARA VERIFICAÇÃO DE SOLVÊNCIA NAS EMPRESAS OI S/A E TIM S/A.

Flavio Heleno Solano Reis, Petrus Fabiano Araújo de Oliveira, Josy Hellen Azevedo Braga, Milton Pinheiro de Almeida Netto, Mayla Rodrigues Barbosa Cunha

A empresa TIM S/A operou no período de 2014 a 2018 de maneira mais equilibrada, tendo um leve crescimento de 2014 a 2016, partindo de um fator de solvência de 3,00 em 2014 e chegando a um fator de 3,34. Após isso no ano seguinte a empresa decai de 3,34 para 2,52 em 2017 e mais um pouco chegando em 2018 com o valor de 2,24, mas conseguindo ficar na região de solvência, honrando seus compromissos, conforme aponta o fator de insolvência.

O termômetro de Kanitz foi de fundamental importância para esta pesquisa, que procurou detalhar o modo como foi criado pelo professor Sthepen Charles Kanitz, como ele utilizou nas empresas que faliram na época, e conseguindo assim uma aceitação do modelo naquele período, sendo utilizado até hoje como ferramenta de gestão por muitos estudiosos e gestores de grandes organizações.

Diante dos resultados demonstrados e das respectivas análises é possível afirmar que perante os resultados obtidos dos dados contábeis das empresas, e foi possível uma análise profunda de seus demonstrativos, possibilitando checar cada ponto de elevação ou queda das empresas e os respectivos anos de sua ocorrência, gerando assim um gráfico para cada empresa para ser utilizado para acompanhamento e gestão das mesmas.

Assim recomendam se novos estudos mais aprofundados e com utilização de outras técnicas e ferramentas de gestão para que possam ser utilizados nas empresas citadas e em outras empresas conforme o desejo de seus gestores, uma vez que tem se tornado de suma importância o acompanhamento das empresas para uma possível previsão de futuro das mesmas a partir de dados passados, pois com essa preocupação poderemos ter no futuro empresas melhores em sua economia e demonstrativos financeiros, favorecendo assim, o crescimento da economia do país.

\section{REFERÊNCIAS}

ALMEIDA, Marcelo Cavalcanti. Análise das demonstrações contábeis em IFRS e CPC: facilitada e sistematizada. São Paulo: Atlas, 2019.

BOVESPA. Balanço Financeiro Oi - OIBR4. São Paulo: [S.e], 2019a. Disponível em: https://br.advfn.com/bolsa-de-valores/bovespa/oi-OIBR4/balanco/consolidado. Acesso em: 14 set. 2019.

BOVESPA. Balanço Financeiro Tim - TIMP3. São Paulo: [S.e], 2019b. Disponível em: https://br.advfn.com/bolsa-de-valores/bovespa/tim-TIMP3/balanco/consolidado. Acesso em: 14 set. 2019.

CREPALDI, Silvio. Contabilidade financeira e gerencial. Catho Online - Material de Apoio do Curso Online Contabilidade Financeira e Gerencial. 2006. Disponível em: https://www.catho.com.br/curso/elearning/biblioteca/contabilidade/textodeapoio.pdf. Acesso em: 29 set. 2019.

DINIZ, Natália. Analise das Demonstrações Financeiras. Rio de Janeiro: Editora SESES, 2015. 


\section{RECIMA21 - REVISTA CIENTÍFICA MULTIDISCIPLINAR}

APLICAÇÃO DO MÉTODO KANITZ PARA VERIFICAÇ̃̃O DE SOLVÊNCIA NAS EMPRESAS OI S/A E TIM S/A

Flavio Heleno Solano Reis, Petrus Fabiano Araújo de Oliveira, Josy Hellen Azevedo Braga, Milton Pinheiro de Almeida Netto, Mayla Rodrigues Barbosa Cunha

FARIAS FILHO, Milton Cordeiro; ARRUDA FILHO, Emílio J. M. Planejamento da Pesquisa Cientifica. 2. ed. São Paulo: Atlas, 2015.

GIL, Antônio Carlos. Como Elaborar Projetos de Pesquisa. 4. ed. São Paulo: Atlas, 2002.

IUDÍCIBUS, Sérgio. Contabilidade Gerencial. 6. ed. São Paulo: Atlas, 1998.

KASSAI, J.; KASSAI, S. Desvendando o termômetro de insolvência de Kanitz. 2003. Disponível em: http://www.anpad.org.br/admin/pdf/enanpad1998-ccg-08.pdf. Acesso em: 05 set. 2019.

MARION, José Carlos. Análise das Demonstrações Contábeis. 8. ed. São Paulo: Atlas, 2019.

MARION, José Carlos. Análise das Demonstrações Contábeis: Contabilidade Empresarial. 3. ed. São Paulo: Atlas, 2006.

MARION, José Carlos. Contabilidade Empresarial. 9. ed. São Paulo: Atlas, 2002.

OI. Relatórios Anuais Ol. Rio de Janeiro. Disponível em: https://www.oi.com.br/ri/conteudo pt.asp?idioma=0\&conta=28\&tipo=43589. Acesso em: 29 set. 2019.

OLIVEIRA, Petrus. Analise de Insolvência. 2019. Apostila do curso de Bacharelado em Ciências Contábeis- Escola Superior Madre Celeste - Esmac. Belém: Esmac, 2019.

TIM. Demonstrações Financeiras TIM. Rio de Janeiro. Disponível em: https://ri.tim.com.br/ListResultados/Central-de-Resultados?=8aFYrvuD6r3eo9YseDEvrw==. Acesso em: 29 set. 2019.

WALD. Recuperação judicial oi. São Paulo. Disponível em: http://www.recuperacaojudicialoi.com.brl. Acesso em: 25 fev. 2020. 